\title{
A detailed calculation of neutral hydrogen ionization frequencies used in turbulence transport models in the heliosphere (Research Note)
}

\author{
N. E. Engelbrecht ${ }^{1,2}$ and R. D. Strauss $s^{2,3}$ \\ ${ }^{1}$ South African National Space Agency, 7200 Hermanus, South Africa \\ e-mail: $n$. eugene.engelbrecht@gmail.com \\ 2 Center for Space Research, North-West University, 2790 Potchefstroom, South Africa \\ e-mail: 12580996@nwu.ac.za \\ ${ }^{3}$ On sabbatical leave at the Center for Space Plasma and Aeronomic Research, University of Alabama in Huntsville, Huntsville, \\ AL 3585, USA
}

Received 9 March 2015 / Accepted 7 June 2015

\begin{abstract}
It is generally accepted that the solar wind is significantly heated beyond $\sim 10$ AU by the turbulent decay of pickup ion generated Alfvénic fluctuations. Here, we present a detailed and general calculation of the pickup ion ionization frequencies, and we evaluate these quantities within the solar wind termination shock along the stagnation line. For this supersonic solar wind region, inside of the solar wind termination shock, our results compare well with earlier estimates of these frequencies. When, in the future, turbulence transport models are extended into the heliosheath, the methodology outlined in this paper can be used to calculate ionization frequencies in this hot and dense plasma region where the resulting calculations become more complex.
\end{abstract}

Key words. turbulence - Sun: heliosphere - solar wind

\section{Introduction}

The radial profile of the solar wind proton temperature has long been known to deviate significantly from the dependence expected of a purely adiabatic expansion (see, e.g., Gazis et al. 1994; Richardson et al. 1995). Overall, Richardson et al. (1995) report a $r^{-0.49 \pm 0.01}$ dependence for the solar wind proton temperature, quite different from the $r^{-4 / 3}$ dependence expected of purely adiabatic expansion. Smith et al. (2001) argue that this effect is not related to the solar cycle. One possible explanation, proposed by Richardson et al. (1995), for the increase in temperature at the largest radial distances is that it is a latitudinal effect, as the Voyager spacecraft leaves the ecliptic plane after $\sim 20-30$ AU. This explanation, however, does not fully explain the higher temperatures observed in the ecliptic. A possible source of energy for this phenomenon, suggested by Williams et al. (1995), is the formation of pickup ions from interstellar neutral hydrogen, which could generate turbulence (Lee \& Ip 1987), the cascade of which would then deposit this energy as heat into the solar wind core protons. As neutral hydrogen particles enter the heliosphere, they become ionized either by charge exchange mechanisms with solar wind protons, by solar ultraviolet light, or by electron impacts (see, e.g., Zank 1999; Fahr et al. 2000; Isenberg et al. 2003; Isenberg 2005; Scherer et al. 2014). This introduces an unstable distribution of pickup protons into the solar wind, which in turn are quickly scattered, generating wavemodes propagating parallel to the heliospheric magnetic field (Smith et al. 2001). Any turbulence transport model that only includes 2D-like fluctuations cannot, therefore, self-consistently include the effect of these pickup ions. Only a fraction of the pickup ion's energy is deposited in this way (Isenberg et al. 2003; Isenberg 2005), but the energy contributed is significant enough to make this a viable heating mechanism (Isenberg 2005). This has indeed been demonstrated in many studies, where increasingly sophisticated turbulence transport models are employed to model the effects of this cascade on the solar wind proton temperature profile (see, e.g., Zank et al. 1996; Smith et al. 2001; Isenberg 2005; Breech et al. 2008; $\mathrm{Ng}$ et al. 2010; Oughton et al. 2011; Adhikari et al. 2014, and other references below). The purpose of this research note is to show that the relatively simple means by which the above mentioned studies model the ionization frequency of the incoming interstellar hydrogen in the supersonic solar wind is indeed accurate, and does not significantly deviate from the results of a calculation of the same from first principles.

In turbulence transport models the energy contribution due to the turbulent fluctuation energy generated by the formation of pickup ions is generally modeled using an expression of the form (Williams \& Zank 1994)

$\dot{E}_{\mathrm{PI}}=f \frac{v_{\mathrm{A}} v_{\mathrm{b}}^{\mathrm{p}}}{n^{\mathrm{p}}} \frac{\mathrm{d} N^{\mathrm{PUI}}}{\mathrm{d} t}=f \frac{v_{\mathrm{A}} v_{\mathrm{b}}^{\mathrm{p}}}{n^{\mathrm{p}}} \frac{N^{\mathrm{H}}}{\tau^{\text {ion }}}=f \frac{v_{\mathrm{A}} v_{\mathrm{b}}^{\mathrm{p}}}{n^{\mathrm{p}}} v^{\text {ion }} N^{\mathrm{H}}$,

where $v_{\mathrm{A}}$ is the Alfvén speed, $v_{\mathrm{b}}^{\mathrm{p}}$ the solar wind proton bulk speed, $N^{\mathrm{PUI}}$ the pickup ion density, $N^{\mathrm{H}}$ the neutral hydrogen density, $n^{\mathrm{p}}$ the solar wind proton density, $\tau^{\text {ion }}$ the ionization time, $v^{\text {ion }}$ the corresponding ionization frequency, and $f$ a scaling factor related to the amount of the pickup ion's energy that is contributed to fluctuations. The formalism used by, e.g., Isenberg (2005), $\mathrm{Ng}$ et al. (2010), Oughton et al. (2011), Engelbrecht \& Burger (2013), $f=\zeta$, which in turn is related to the quantity $f_{D}$ used by, e.g., Williams \& Zank (1994), Smith et al. (2001, 2006), Breech et al. (2008), Usmanov et al. (2012), with $f_{D}=\left(V_{s w} / V_{\mathrm{A}}\right) \zeta$. The quantity $\zeta$ is found from first principles by Isenberg et al. (2003) 

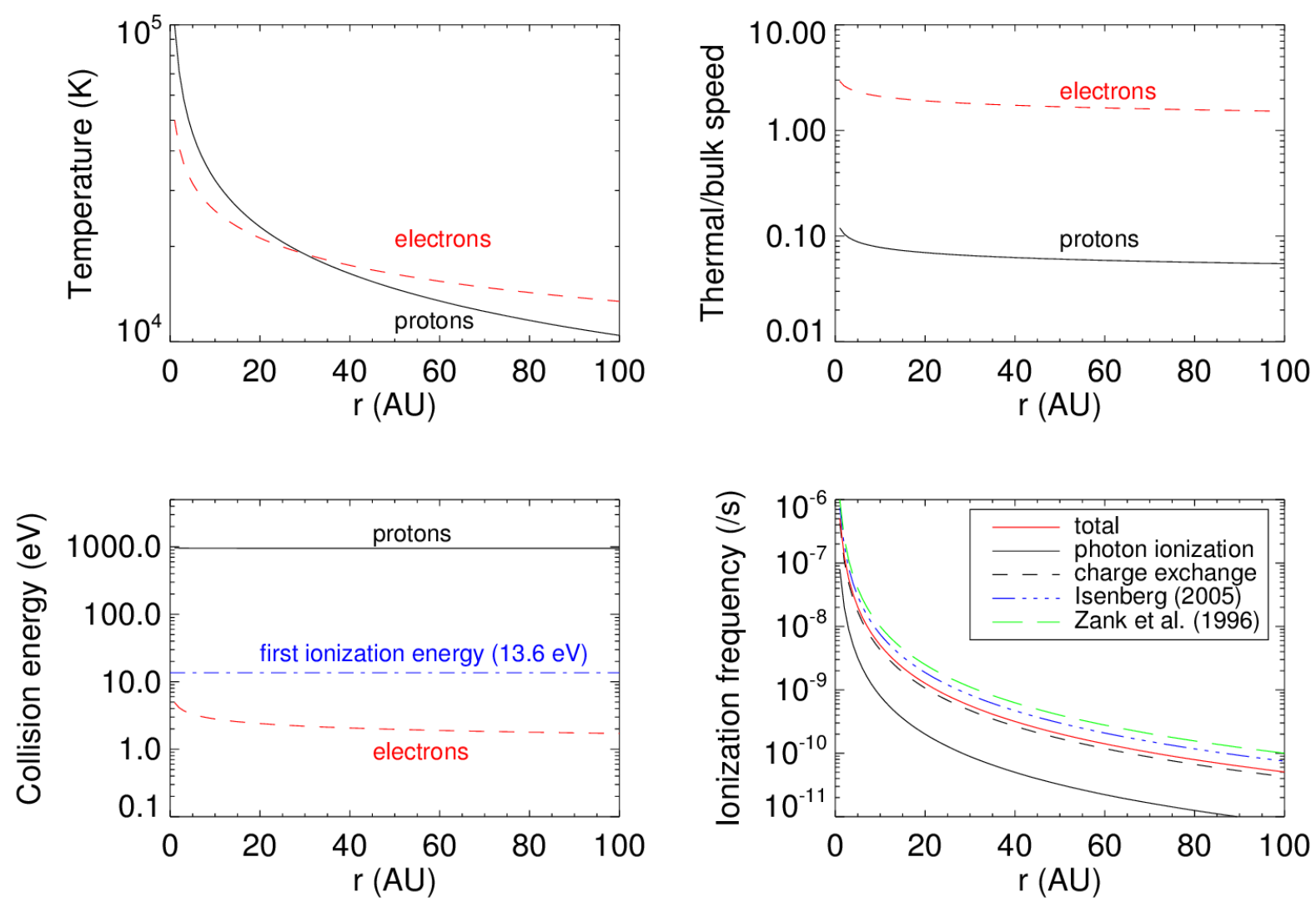

Fig. 1. Radial dependence of the temperature, ratio of the thermal and bulk components of the collision speed, and resulting collision energy for protons and electrons. The bottom right panel shows the calculated ionization frequencies, compared to previous estimates.

and Isenberg (2005) to be a few percent. In Oughton et al. (2011) and Engelbrecht $\&$ Burger (2013), for instance, $\zeta$ is assumed to be constant and equal to 0.04 . This quantity, however, does vary throughout the heliosphere (see, e.g., Isenberg 2005; $\mathrm{Ng}$ et al. 2010). The neutral hydrogen density is usually modeled within the termination shock with

$N^{\mathrm{H}}=N_{\mathrm{TS}}^{\mathrm{H}} \exp \left[-\frac{L_{\mathrm{cav}} / r}{\theta_{n} / \sin \theta_{n}}\right]$,

where $N_{\mathrm{TS}}^{\mathrm{H}}$ is the neutral hydrogen density at the termination shock, $L_{\text {cav }}$ the radial heliocentric extent of the ionization cavity, and $\theta_{n}$ the angle measured between an observation point and the neutral hydrogen upstream direction. A value of $0.1 / \mathrm{cc}$ is usually chosen for $N_{\mathrm{TS}}^{\mathrm{H}}$, as for example by Breech et al. (2008), Oughton et al. (2011), following the results of line-of-sight observations of nearby stellar Lyman- $\alpha$ emission lines (see, e.g., Anderson et al. 1978; Gry \& Jenkins 2001). Various Lyman- $\alpha$ observations from other studies indicate that this parameter varies within a range of about $0.05-0.3 / \mathrm{cc}$ (Scherer et al. 1999), but Bzowski et al. (2009) report an observational value of $0.09 \pm 0.022 / \mathrm{cc}$ for this quantity, consolidated from measurements taken by various spacecraft. The ionization cavity size $L_{\text {cav }}$ appears dependent upon the solar cycle (Smith et al. 2001), and two values are commonly used as inputs for turbulence transport models: $L_{\text {cav }}=5.6 \mathrm{AU}$ (see, e.g., Isenberg et al. 2003; Isenberg 2005; Isenberg et al. 2010; Oughton et al. 2011), and $L_{\mathrm{cav}}=8 \mathrm{AU}$ (see, e.g., Smith et al. 2001; Breech et al. 2008). Schwadron \& McComas (2010), however, state that most incoming neutral hydrogen atoms would be ionized within $\sim 4 \mathrm{AU}$. A value of $0^{\circ}$ is generally assumed for $\theta_{n}$, thereby implying a spatially uniform influx of neutral hydrogen (see, e.g., Smith et al. 2001; Breech et al. 2008; Pei et al. 2010; Oughton et al. 2011). The above approach assumes a cold neutral hydrogen density (Vasyliunas \& Siscoe 1976), which may not be the best choice of model (see, e.g., Zank 1999), and is not the most realistic of scenarios given recent IBEX results (see, e.g., Fuselier et al. 2009). This model, however, has the benefit of being relatively tractable and has yielded good agreement with solar wind proton temperature observations when used in conjunction with turbulence transport models (see, e.g., Smith et al. 2001, 2006; Isenberg 2005; Isenberg et al. 2010; $\mathrm{Ng}$ et al. 2010; Oughton et al. 2011; Adhikari et al. 2014).

\section{Ionization frequencies}

Generally, the various turbulence transport studies cited above assume that the interstellar neutral hydrogen ionization frequency scales as $r^{-2}$, and that the formation of pickup ions is governed by photoionization or charge exchange with solar wind protons. As this radial dependence cancels neatly with that of the solar wind proton density in Eq. (1), only the $1 \mathrm{AU}$ value of this quantity (and of the proton density) is employed in this expression. Two different values for the ionization frequency at Earth are generally employed: a value of $10^{-6} \mathrm{~s}^{-1}$ by, e.g., Zank et al. (1996), Smith et al. (2001) and Breech et al. (2008), and a more commonly used value of $7.5 \times 10^{-7} \mathrm{~s}^{-1}$ by, e.g., Isenberg et al. (2003), Isenberg (2005), Smith et al. (2006) and Oughton et al. (2011). This approach does not take into account a solar cycle dependence of this quantity, the possibility of which being suggested by Smith et al. (2001). In what follows, the former value is referred to as the Zank et al. (1996) value, while the latter is referred to as the Isenberg et al. (2003) value. These two ionization time scalings are shown as function of heliocentric radial distance along the stagnation line in the bottom panel of Fig. 1. 
We consider three ionization processes, respectively photoionization (pi), charge exchange (ce), and electron impact (ei), where

$$
\begin{aligned}
& \text { pi: } \mathrm{H}+\gamma \rightarrow p^{\text {PUI }} \\
& \text { ce } p+\mathrm{H} \rightarrow \mathrm{H}+p^{\text {PUI }} \\
& \text { ei: } \mathrm{H}+\mathrm{e}^{-} \rightarrow p^{\text {PUI }}+2 \mathrm{e}^{-} .
\end{aligned}
$$

The total ionization frequency can then be written as the sum of the ionization frequencies due to each process, such that

$v^{\text {ion }}=v^{\mathrm{pi}}+v^{\mathrm{ce}}+v^{\mathrm{ei}}$.

In general, the ionization frequency can be written as

$v^{i}=n^{j} \sigma^{i}\left(v_{\mathrm{coll}}^{i}\right) v_{\mathrm{coll}}^{i}$,

where $i \in\{$ pi,ce, ei $\}$ describes the different processes, $\sigma^{i}$ the corresponding cross-sections, $n^{j}$ the number density of the different particle species interacting with neutral hydrogen (with $j \in\{\gamma, p, e\})$ and $v_{\text {coll }}^{i}$ is the different collision speed for every process. The collision speed can be written in a general form as

$v_{\text {coll }}^{i}=\sqrt{\left(v_{\text {therm }}^{j}+v_{\text {therm }}^{\mathrm{H}}\right)^{2}+\left(\boldsymbol{v}_{\mathrm{b}}^{j}-\boldsymbol{v}_{\mathrm{b}}^{\mathrm{H}}\right)^{2}}$,

although this is, of course, not valid for photoionization. Here $v_{\text {therm }}^{j}$ and $\boldsymbol{v}_{\mathrm{b}}^{j}$ are the thermal speed and bulk velocity respectively, with

$v_{\text {therm }}^{j}=\sqrt{\frac{2 k_{\mathrm{B}} T^{j}}{m_{j}}}$ and $v_{\text {therm }}^{\mathrm{H}}=\sqrt{\frac{2 k_{\mathrm{B}} T^{\mathrm{H}}}{m_{\mathrm{H}}}}$.

When only considering the stagnation line, $\boldsymbol{v}_{\mathrm{b}}^{j}=v_{\mathrm{b}}^{j} \hat{\boldsymbol{r}}$ and $\boldsymbol{v}_{\mathrm{b}}^{\mathrm{H}}=$ $-v_{\mathrm{b}}^{\mathrm{H}} \hat{\boldsymbol{r}}$, so that $v_{\text {coll }}^{i}$ reduces to

$v_{\text {coll }}^{i}=\sqrt{\left(v_{\text {therm }}^{j}+v_{\text {therm }}^{\mathrm{H}}\right)^{2}+\left(v_{\mathrm{b}}^{j}+v_{\mathrm{b}}^{\mathrm{H}}\right)^{2}}$.

When the charge-exchange ionization process is considered, the ionization frequency can be written as

$v^{\mathrm{ce}}=n^{\mathrm{p}} \sigma^{\mathrm{ce}}\left(v_{\text {coll }}^{\mathrm{ce}}\right) v_{\text {coll }}^{\mathrm{ce}}$,

where the proton number density, normalized to a value $n_{0}^{\mathrm{p}}$ at Earth taken here to be $5 / \mathrm{cc}$ (consistent with the reported observational range of 5-10/cc Wang et al. 2007), is given by $n^{\mathrm{p}}=$ $n_{0}^{\mathrm{p}}\left(r_{0} / r\right)^{2}$. We use a value of $v_{\mathrm{b}}^{\mathrm{H}}=26 \mathrm{~km} \mathrm{~s}^{-1}$ and $T^{\mathrm{H}}=6300 \mathrm{~K}$ for the hydrogen bulk speed and temperature, as reported by McComas et al. (2015). For the solar wind protons, we assume the typical ecliptic value of $v_{\mathrm{b}}^{\mathrm{p}}=400 \mathrm{~km} \mathrm{~s}^{-1}$, with a temperature that decreases as $T^{\mathrm{p}} \sim r^{-0.49}$ after Richardson et al. (1995), normalized to $10^{5} \mathrm{~K}$ at Earth (see, e.g., Smith et al. 2001). The top left panel of Fig. 1 shows the assumed proton temperature profile and the top right panel the ratio of the thermal $\left(v_{\text {therm }}^{\mathrm{p}}+v_{\text {therm }}^{\mathrm{H}}\right)$ to the bulk speeds $\left(v_{\mathrm{b}}^{\mathrm{p}}+v_{\mathrm{b}}^{\mathrm{H}}\right)$ that enter the calculation of $v_{\text {coll }}^{\mathrm{ce}}$. It is clear that, for charge exchange, the bulk speed is the dominant contribution to the collision speed. Beyond the termination shock, however, the plasma becomes slower, denser, and hotter, so that the thermal component may become significant in this region. The relative collision energy, needed to evaluate $\sigma^{i}\left(E_{\text {coll }}^{i}\right)$, is calculated as $E_{\text {coll }}^{i}=m_{j}\left(v_{\text {coll }}^{i}\right)^{2} / 2$ (see the bottom left panel of Fig. 1). The cross-section we use for this particular interaction is reported by Scherer et al. (2014), with a value of $\sim 2 \times 10^{-15} \mathrm{~cm}^{-2}$ taken for a relative collision energy of $\sim 930 \mathrm{eV}$. This value is identical to that employed by Usmanov et al. (2012). The resulting radial dependence of the charge exchange ionization frequency is shown in the bottom right panel of Fig. 1. so that

For photoionization, $v_{\text {coll }}^{\mathrm{pi}}=c$, where $c$ is the speed of light,

$v^{\mathrm{pi}}=\sigma\left(c n^{\gamma}\right)=\sigma \mathcal{F}$.

Moreover, the photon flux decreases as

$\mathcal{F}=\mathcal{F}_{0}\left(\frac{r_{0}}{r}\right)^{2}$

which, following Scherer et al. (2014), leads to $v^{\mathrm{pi}}=8 \times$ $10^{-8}\left(r_{o} / r\right)^{2}$. This is very similar to the 1 AU value for the photoionization frequency of $9 \times 10^{-8} \mathrm{~Hz}$ employed by Whang (1998).

The ionization frequency for electron impacts can be expressed by

$v^{\mathrm{ei}}=n^{\mathrm{e}} \sigma^{\mathrm{ei}}\left(v_{\text {coll }}^{\mathrm{ei}}\right) v_{\text {coll }}^{\mathrm{ei}}$.

We assume the solar wind plasma to be quasi-neutral, $n^{\mathrm{e}} \approx$ $n^{\mathrm{p}}$, and that the different species are co-moving, $v_{\mathrm{b}}^{\mathrm{e}} \approx v_{\mathrm{b}}^{\mathrm{p}}$. Observations indicate that solar wind protons and electrons are not in thermal equilibrium; Cranmer et al. (2009) show that $T^{\mathrm{e}}$ is 2-3 times lower than $T^{\mathrm{p}}$ at Earth. Moreover, Sittler \& Scudder (1980) find that $T^{\mathrm{e}}$ shows near isothermal behavior. We therefore assume $T^{\mathrm{e}} \sim r^{-2 / 7}$, normalized to $5 \times 10^{4} \mathrm{~K}$ at Earth. The radial dependence of $T^{\mathrm{e}}$ is, because of the limited number of observations at larger radial distances, relatively unknown, and our estimates can probably be considered an upper limit (e.g., Landi et al. 2012). The top panels of Fig. 1 once again show the assumed $T^{\mathrm{e}}$ profile and the ratio of thermal to bulk speed for the electron impact collision speed. As opposed to protons, the electron thermal component dominates $v_{\mathrm{coll}}^{\mathrm{ei}}$. Again calculating $E_{\mathrm{ei}}^{\mathrm{coll}}$, shown in the bottom left panel, we find $E_{\mathrm{ei}}^{\text {coll }}<13.6 \mathrm{eV}$, which means that for the assumed set of parameters, neutral hydrogen cannot be ionized through electron impacts, i.e., $v^{\text {ei }} \approx 0$. If, however, electrons are sufficiently heated beyond the terminations shock, as suggested by Chashei \& Fahr (2014), the electron impact ionization process may become effective in the heliosheath.

Figure 1 shows the ionization frequencies due to each of the processes discussed above (bottom right panel) as well as the total ionization frequency thus calculated, as function of radial distance. The total ionization frequency calculated (red line) shows a $r^{-2}$ dependence, with a value of $5.07 \times 10^{-7} \mathrm{~s}^{-1}$ at 1 AU, corresponding to an ionization time at Earth of $\tau_{\mathrm{o}}^{\text {ion }} \approx 1.97 \times 10^{6} \mathrm{~s}$. It is clear that electron impact cannot contribute to the total ionization frequency, and that charge exchange contributes most. The total calculated ionization frequency differs very little from the previously assumed scalings of the same (green and blue lines) in the very inner (within $\sim 20 \mathrm{AU}$ ) heliosphere. The differences, however, become larger with increasing radial distance, so that at $100 \mathrm{AU}$ the calculated total ionization frequency is $\sim 97 \%$ smaller than the Zank et al. (1996) value, and $\sim 48 \%$ smaller than the Isenberg et al. (2003) value.

Figure 2 shows radial solar wind proton temperature profiles calculated with the two-component turbulence transport model proposed by Oughton et al. (2011), following the approach of Engelbrecht \& Burger (2013), for the various ionization frequencies discussed above. The boundary value of the solar wind proton temperature at $0.3 \mathrm{AU}$ used here is $4 \times 10^{5} \mathrm{~K}$ so as to ensure a temperature at Earth of $10^{5} \mathrm{~K}$, while all other boundary values for turbulence quantities are held at the same values used by Engelbrecht \& Burger (2013). Voyager observations as reported by Smith et al. (2001) are also shown, to guide the eye, 


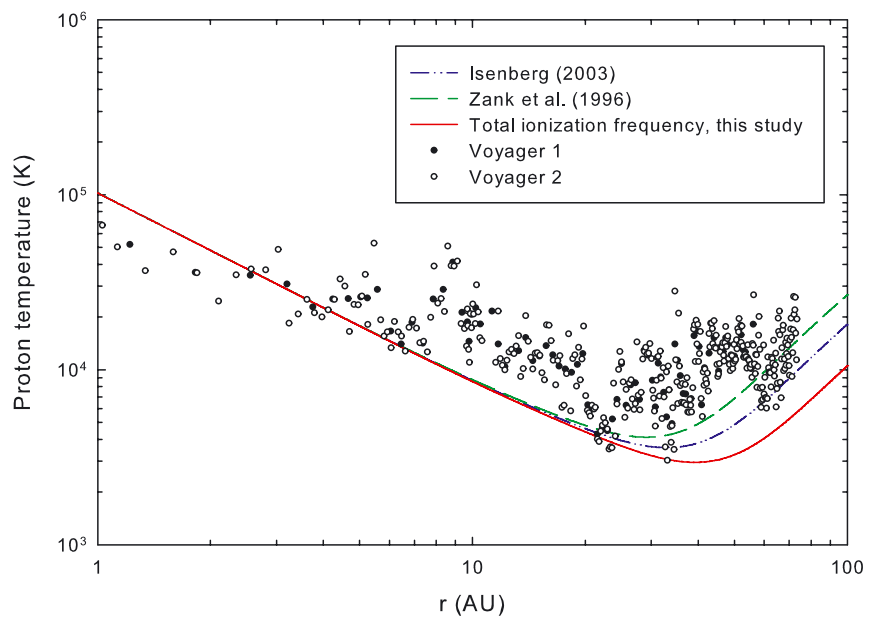

Fig. 2. Solar wind proton temperatures calculated using the Oughton et al. (2011) turbulence transport model using various ionization frequencies, as function of heliocentric radial distance. Voyager data shown are taken from Smith et al. (2001).

as no attempt at data fitting is made. However, the model solutions broadly follow their radial trend. In the very inner heliosphere (within $\sim 10 \mathrm{AU}$ ), the temperature profiles for each of the frequencies are almost identical, as expected from Fig. 1. At larger radial distances, the differences in ionization frequencies employed are clearly reflected in computed temperature profiles, with the largest ionization frequencies (in this case that used by Zank et al. 1996) leading to the largest temperatures at $100 \mathrm{AU}$. At $100 \mathrm{AU}$, there is an $\sim 72 \%$ difference between results computed using the total ionization frequency that we calculate, and those computed using the Isenberg et al. (2003) ionization frequency, with an $\sim 154 \%$ difference from results calculated using the Zank et al. (1996) ionization frequency.

\section{Concluding remarks}

Careful calculation of the ionization frequency of incoming interstellar neutral hydrogen, taking the processes of photoionization, charge exchange, and electron impact into account, leads to a result not vastly different from the approach usually taken when this quantity is modeled in turbulence transport studies, with a difference of $\sim 48 \%$ when compared with the commonly used value of Isenberg et al. (2003). Radial solar wind proton temperature profiles calculated using the different ionization frequencies discussed above reflect the differences in the frequencies themselves. When compared, the differences in proton temperature profiles calculated with the various ionization frequencies calculated here are large. The profile calculated using the more commonly employed Isenberg et al. (2003) ionization frequency differs from that computed using the ionization frequency calculated here by $\sim 72 \%$ at $100 \mathrm{AU}$, and even though this difference is small relative to the variations in the Voyager observations shown in Fig. 2, it can be significant when careful comparisons of model results with observations need to be made. The collision speed seems too low for electron impact to a be a viable ionization process inside the termination shock regions. Should turbulence transport models be extended into the heliosheath, as in theory has recently become possible with the model proposed by Zank et al. (2012), the heating of the plasma at the termination shock would necessitate a calculation of the ionization frequency along the lines that we present here. Additionally, we note that $v^{\text {ion }}$ will have an azimuthal dependence if calculated throughout the heliosphere, even in the supersonic solar wind (note the vector notation in Eq. (8)) and that this quantity will exhibit solar cycle variations (through the input parameters, e.g., $T^{j}$ and $n^{j}$ ) if calculated time dependently.

Acknowledgements. This work is based on the research supported in part by the National Research Foundation (NRF) of South Africa (RDS is supported through the Thuthuka Program; Grant No. 87998). Opinions expressed and conclusions arrived at are those of the authors and are not necessarily to be attributed to the NRF. RDS acknowledges partial financial support from the Fulbright Visiting Scholar Program. The authors are grateful to Klaus Scherer for various discussions regarding the subject matter presented here. The authors are extremely grateful for the valuable input from the anonymous reviewer.

\section{References}

Adhikari, L., Zank, G. P., Hu, Q., \& Dosch, A. 2014, ApJ, 793, 52 Anderson, R. C., Henry, R. C., Moos, H. W., \& Linsky, J. L. 1978, ApJ, 226, 883 Breech, B., Matthaeus, W. H., Minnie, J., et al. 2008, J. Geophys. Res., 113, 8105

Chashei, I. V., \& Fahr, H.-J. 2014, Sol. Phys., 289, 1359

Cranmer, S. R., Matthaeus, W. H., Breech, B. A., et al. 2009, ApJ, 702, 1604

Bzowski, M., Möbius, E., Tarnopolski, S., Izmodenov, V., \& Gloeckler, G. 2009, Space Sci. Rev., 143, 177

Engelbrecht, N. E., \& Burger, R. A. 2013, ApJ, 772, 46

Fahr, H. J., Kausch, T., \& Scherer, H. 2000, A\&A, 357, 268

Fuselier, S., Allegrini, F., Funsten, H. O., et al. 2009, Science, 326, 962

Hunana, P., \& Zank, G. P. 2010, ApJ, 718, 148

Gazis, P. R., Barnes, A., Mihalov, J. D., \& Lazarus, A. J. 1994, J. Geophys. Res., 99,6561

Gry, C., \& Jenkins, E. B. 2001, A\&A, 367, 617

Isenberg, P. A. 2005, ApJ, 623, 502

Isenberg, P. A., Smith, C. W., \& Matthaeus, W. H. 2003, ApJ, 592, 564

Isenberg, P. A., Smith, C. W., Matthaeus, W. H., \& Richardson, J. D. 2010, ApJ, 719,716

Landi, S., Matteini, L., \& Pantellini, F. 2012, ApJ, 760, 143

Lee, M. A., \& Ip, W. H. 1987, J. Geophys. Res., 92, 11041

Lindsay, B. G., \& Stebbings, R. F. 2005, J. Geophys. Res., 110, 12213

McComas, D. J., \& Schwadron, N. A. 2010, ApJ, 712, L157

McComas, D. J., Bzowski, M., Frisch, P., et al. 2015, ApJ, 801, 28

Möbius, E., Bochsler, P., Bzowski, M., et al. 2012, ApJS, 198, 11

Ng, C. S., Bhattacharjee, A., Munsi, D., Isenberg, P. A., \& Smith, C. W. 2010, J. Geophys. Res., 115, 2101

Oughton, S., Matthaeus, W. H., \& Dmitruk, P. 2006, Phys. Plasmas, 13, 042306 Oughton, S., Matthaeus, W. H., Smith, C. W., Bieber, J. W., \& Isenberg, P. A. 2011, J. Geophys. Res., 116, 8105

Pei, C., Bieber, J. W., Breech, B., et al. 2010, J. Geophys. Res., 115, 3103

Richardson, J. D., Paularena, K. I., Lazarus, A. J., \& Belcher, J. W. 1995, Geophys. Res. Lett., 22, 325

Sittler, E. C., \& Scudder, J. D. 1980, J. Geophys. Res., 85, 5131

Scherer, H., Bzowski, M., Fahr, H.-J., \& Rucinski, D. 1999, A\&A, 342, 601

Scherer, K., Fichtner, H., Fahr, H.-J., Bzowski, M., \& Ferreira, S. E. S. 2014, A\&A, 563, A69

Schwadron, N. A., \& McComas, D. J. 2010, ApJ, 712, L157

Smith, W. S., Matthaeus, W. H., Zank, G. P., et al. 2001, J. Geophys. Res., 106, 8253

Smith, C. W., Isenberg, P. A., Matthaeus, W. H., \& Richardson, J. D. 2006, ApJ, 638,508

Usmanov, A. V., Goldstein, M. L., \& Matthaeus, W. H. 2012, ApJ, 754, 40

Vasyliunas, V. M., \& Siscoe, G. L. 1976, J. Geophys. Res., 81, 1247

Wang, C. P., Lyons, L. R., Nagai, T., Weygand, J. M., \& McEntire, R. W. 2007, J. Geophys. Res., 112, 10224

Whang, Y. C. 1998, J. Geophys. Res., 103, 17419

Williams, L. L., \& Zank, G. P. 1994, J. Geophys. Res., 99, 19229

Williams, L. L., Zank, G. P., \& Matthaeus, W. H. 1995, J. Geophys. Res., 100, 17059

Zank, G. P. 1999, Space Sci. Rev., 89, 413

Zank, G. P., Matthaeus, W. H., \& Smith, C. W. 1996, J. Geophys. Res., 101, 17093

Zank, G. P., Dosch, A., Hunana, P., et al. 2012, ApJ, 745, 35 